\title{
Types of sedation used in pediatric dentistry
}

\author{
Hanan Raja Aljohani ${ }^{1 *}$, Rinad Abdulmonam Albalawi ${ }^{2}$, Fajr Adel Orri ${ }^{3}$, \\ Khalid Mubarak Alshammari ${ }^{4}$, Narmeen Abdulmannan Shikdar ${ }^{5}$, Bader Hussain Al Sayhab ${ }^{6}$, \\ Hifa Mohammed Alkahtani ${ }^{7}$, Alanoud Ali Alwably ${ }^{8}$, Alanoud Mohammed Algazlan ${ }^{8}$, \\ Ali Abdullah Alghamdi ${ }^{9}$, Albandari Menahi Alqahtani ${ }^{10}$
}

\author{
${ }^{1}$ Department of Pediatric Dentistry, East Jeddah Hospital, Jeddah, Saudi Arabia \\ ${ }^{2}$ General Dentist, Ministry of Health, Riyadh, Saudi Arabia \\ ${ }^{3}$ College of Dentistry, October University, Cairo, Egypt \\ ${ }^{4}$ General Dentist, Ministry of Health, Hail, Saudi Arabia \\ ${ }^{5}$ College of Dentistry, King Abdulaziz University, Jeddah, Saudi Arabia \\ ${ }^{6}$ College of Dentistry, Najran University, Najran, Saudi Arabia \\ ${ }^{7}$ Dental Department, Al-Kharj Military Industrial Corporation Hospital, Al-Kharj, Saudi Arabia \\ ${ }^{8}$ College of Dentistry, Princess Nourah Bint Abdul Rahman University, Riyadh, Saudi Arabia \\ ${ }^{9}$ General Dentist, Al Jawdah Dental Clinics, Jeddah, Saudi Arabia \\ ${ }^{10}$ College of Dentistry, Imam Abdulrahman Bin Faisal University, Dammam, Saudi Arabia
}

Received: 15 November 2021

Accepted: 29 November 2021

\author{
*Correspondence: \\ Dr. Hanan Raja Aljohani, \\ E-mail: Hananaljohani99@gmail.com
}

Copyright: (c) the author(s), publisher and licensee Medip Academy. This is an open-access article distributed under the terms of the Creative Commons Attribution Non-Commercial License, which permits unrestricted non-commercial use, distribution, and reproduction in any medium, provided the original work is properly cited.

\begin{abstract}
Childhood dental anxiety usually results from painful and frightening dental traumas. Accordingly, children might want to avoid dental treatment if not adequate precautions were not taken during these settings. Thus, sedation is being widely used in different dental settings with favorable outcomes. In the present literature review, we have discussed sedation's different types and approaches in pediatric dentistry settings. We also reviewed the main medications that can be administered in these settings, together with the potential routes of administration, safety and contraindications. As a result, the pediatric dentist should identify patients suitable for sedation, choose the right type of sedation, prepare the media for successful procedures, and select the proper medication. Careful monitoring and adequate preparation are also critical to enhance the prognosis and manage the potential complications. Eventually, sedation might improve the compliance of pediatric patients and reduce the dilemma of fear from painful dental procedures. This has been associated with favorable outcomes for the dentist, as well. Therefore, further research is needed for additional validation and innovation in this field.
\end{abstract}

Keywords: Sedation, Pediatrics, Dentistry, Anesthesia, Conscious sedation

\section{INTRODUCTION}

Dental procedures might be challenging in pediatric settings due to the reduced coping skills of children, requiring a specific quality of care and management. Psychological harm might result from an unpleasant dental experience. ${ }^{1-3}$ This effect has been estimated to be worse than pain and discomfort that might result from an underlying dental disorder. Childhood dental anxiety usually results from painful and frightening dental traumas. Accordingly, children might want to avoid dental treatment if not adequate precautions were not taken during these settings. ${ }^{4}$

The reported fear usually persists during adulthood. Estimates showed that avoiding essential dental care was prevalent in $10-20 \%$ of the United States adult 
population. ${ }^{5,6}$ Accordingly, sedation has been proposed as an ideal approach to reduce these harmful events and increase trust between the child, dentist and family.,8 Thus, sedation is being widely used in different dental settings with favorable outcomes. Different types of sedation have been proposed in the literature for pediatric dental settings. ${ }^{9}$ In the present literature review, we aimed to discuss these types. Besides, also discussed the different medications commonly used to induce sedation in children, together with their safety profiles and potential complications.

\section{METHODS}

This literature review was based on an extensive literature search in Medline, Cochrane and EMBASE databases which was performed on 2nd October 2021 using the medical subject headings (MeSH) or a combination of all possible related terms, according to the database. To avoid missing poetential studies, a further manual search for papers was done through Google Scholar, while the reference lists of the initially included papers. Studies discussing types of sedation used in pediatric dentistry were screened for useful information, with no limitations posed on date, language, age of participants or publication type.

\section{DISCUSSION}

\section{Overview}

Compared to adults, children usually have a higher need for sedation during different surgical procedures due to their behavioral characteristics. This need was most increased when children reach six months of age and become more aware of the related peri-operative anxiety events. Although anxiety is usual in these events, children have different ways to express this, leading to serious adverse events. Therefore, dentists and clinicians should consider different approaches in this regard to relieving the related anxiety. These approaches usually differ based on the needs of patients, dentists and parents. ${ }^{10}$

\section{Considerations}

According to many dentists and researchers, different considerations should be considered when sedating pediatric patients other than adults because of various reasons. For instance, there were anatomical variations in children including the relatively large tongue, which might contribute to airway obstruction when sedation has been introduced in these patients. Furthermore, in addition to being potentially obscured by the giant tongue, evidence indicated that the larynx was located more anteriorly and at a higher level than in adults. ${ }^{11}$ Subsequently, increased mucus secretion of edema secondary to the induction of sedation can also block the airways in children who usually have small nares. Some of them were even obligate nose breathers. As a result, sedation in children can be significantly associated with more frequent airway obstruction than in adults. In addition, the incidence of hypoxemia is also higher in children due to the relatively reduced functional residual capacity. ${ }^{12}$ Accordingly, dentists should decide the best approach for sedation of children and provide a careful preoperative examination of the airways to enhance the efficacy and safety profile of the process. Finally, the development of bradycardia is a common event following sedation, as indicated by previous studies. Although this effect might not seem severe and could be potentially easily managed, evidence suggested that when bradycardia was present, low cardiac output manifested in the affected children. ${ }^{12,13}$ Thus, in addition to the dominant effect of the vagus nerve, bradycardia in children might be a standard consideration following sedation. However, it should be noted that the administration of preoperative atropine can manage the condition. ${ }^{13,14}$ Nevertheless, these points indicated that dentists should decide the best type for sedation in children after carefully evaluating them preoperatively. ${ }^{12}$ Moreover, evidence showed that the Houpt behavior rating scale can be used to assess the child's behavior, depth of sedation and the total rating of the visit (Figure 1). ${ }^{9,15}$

\begin{tabular}{llll}
\hline Alertness & Crying & Movement & Overall \\
\hline Asleep & No crying & No movement & Excellent: no crying or movement \\
$\begin{array}{l}\text { Drowsy, } \\
\text { disoriented }\end{array}$ & $\begin{array}{l}\text { Intermittent or mild } \\
\text { crying }\end{array}$ & $\begin{array}{l}\text { Controllable, not interfering } \\
\text { with treatment }\end{array}$ & $\begin{array}{l}\text { Very good: some limited crying or } \\
\text { movement }\end{array}$ \\
$\begin{array}{l}\text { Fully awake, } \\
\text { alert }\end{array}$ & $\begin{array}{l}\text { Continuous or } \\
\text { strong crying }\end{array}$ & Continuous, making & Good: difficult, but all treatment was \\
& treatment difficult & performed \\
& Hysterical crying & Violent, interrupting & Fair: treatment interrupted, but \\
& & movement & eventually completed \\
& & & Poor: treatment interrupted, only partial \\
& & & treatment was completed \\
& & & Aborted: no treatment rendered
\end{tabular}

Figure 1: Houpt behavior rating scale. ${ }^{15}$ 


\section{Types}

In this section, we will discuss the different types of sedation that have been described in the literature in pediatric dentistry settings. According to the various studies, evidence showed that different approaches were valid to sedate pediatric patients during dentistry-related procedures. Studies showed that sedation can be minimal, moderate or deep with variable degrees of sedation based on different factors regarding the patient and the underlying process. ${ }^{16}$ In cases of minimal sedation, it has been reported that pediatric patients were usually responsive to typical verbal commands. However, it has been noticed that patients might usually suffer from impaired cognitive and coordination functions in such situations. The main advantages of minimal sedation included achieving the sought outcomes, with relative comfort to the patient, which appeared calm with no associated secondary cardiopulmonary adverse events during the procedure. ${ }^{12,17}$ On the other hand, when approaching patients with moderate sedation, evidence indicated that these patients usually experience consciousness depression and cognitive impairment. Similar to the case with minimal sedation, moderately sedated patients usually purposefully reply to the different verbal commands. Moreover, it should be noted that this can be either done using light tactile stimulation or even alone with no need for such actions.

On the other hand, it has been demonstrated that a purposeful response can not necessarily be obtained using a reflexive withdrawal. The approach has been marked as a productive and safe technique to achieve sedation in the pediatric population. In this context, evidence indicated that dentists can effectively achieve spontaneous ventilation without interventions to keep the airways of the patients patent, with no reported potential adverse events. ${ }^{18}$ During moderate sedation, it has been furtherly reported that cardiovascular and respiratory functions are also maintained with no further complications occurring to the pediatric patients. Severe depression in the levels of consciousness of deeply sedated patients was also reported to be similar to moderate sedation. However, some key differences were also evidenced in the literature. Although it has been demonstrated that patients can purposefully respond secondary to repeated painful stimulatory responses, evidence indicated that deeply sedated pediatric patients cannot be awakened easily. ${ }^{17}$ Ventilatory functions can also be potentially obtained in these situations and further efforts might be needed to keep them functioning at maximal capacity with no complications. Assistance might be required to these patients to keep the airway patent as it has been reported that spontaneous ventilation might not be adequate in these events. However, evidence indicated that cardiovascular functions were usually not affected following deep sedation. Eventually, the type of sedation can also be classified based on the drug's route of administration into oral and intravenous sedation and sedation by nitrous oxide. ${ }^{12,17}$

\section{Medications}

Different medications have been described in the literature as valid drugs that can effectively induce sedation in the different types of sedation for pediatric patients in the dentistry settings. Among the reported medications, nitrous oxide has been described as a potent analgesic and a poor anesthetic medication. Therefore, it was usually administered with other anesthetic medications (for instance, volatile anesthetics and opioids) or in cases of minimal sedation. ${ }^{19}$ The main advantages of this medication included the rapid recovery after administration and immediate onset of action, owing to its high minimum alveolar concentration, reduced irritability and poor solubility in the blood. ${ }^{18}$ However, nitrous oxide usually impacted the functions of different organs. For instance, it was contraindicated in patients with reduced intracranial compliance because it significantly increased cerebral blood flow and associated pressure. In addition, direct myocardial depression was also reported secondary to the administration of nitrous oxide, and therefore, its administration was not recommended for patients with cardiovascular diseases. The main indication of nitrous oxide was mild-tomoderate anxiety. However, there were many other contraindications reported in the literature. These included patients with psychosis, uncooperative patients who cannot afford to breathe through their noses, patients with chronic obstructive pulmonary diseases and nasal obstruction. ${ }^{12,18}$

Opioids, as fentanyl were also reported in the literature to be used for sedation purposes. However, evidence showed that this drug was usually administered in cases of painful and severe procedures. It can be used in combination with another anesthetic medication to provide analgesia. It produced analgesia in a dosedependent manner. Moreover, evidence showed that it had a short duration with a rapid onset and the efficacy has been reported to be higher than morphine by 100 times. Although the drug can produce cardiovascular stability, reports showed that bradycardia might be associated in some cases. Rapid administration of the modality can also lead to glottic or chest wall rigidity. ${ }^{18}$ Furthermore, respiratory depression can occur, but the effect was dose-dependent. Different routes of administration have been reported in the literature. However, careful monitoring of drug administration was necessary to avoid potential complications. ${ }^{12}$ High doses of chloral hydrate were described in the literature as possible anesthetics that can sedate children in dentistry procedures. Its sedative effect was mediated through trichloro ethanol, which acted via inhibitory mechanisms on the central nervous system. The drug has been reported to have a half-life of eight hours. ${ }^{18}$ However, evidence showed that it had mild analgesic effects. Renal or hepatic impairment and cardiovascular diseases were significant contraindications for using chloral hydrate in children. $^{20}$ 
It should be noted that some reports showed that periods of oxygen desaturation and drowsiness were remarkably prolonged with using the modality. Therefore, careful monitoring of the sedated patients should be performed. Deep levels of sedation can affect some patients, in addition to upper airway obstruction. These were considered the most critical side effects reported with using chloral hydrate. Otherwise, the drug was deemed to be safe in such settings. However, recent evidence concluded that repeated high doses of chloral hydrate, usually administered for sedation, can be carcinogenic in some patients. ${ }^{21}$ Accordingly, the liquid and capsule forms were no longer used in the United States. In addition, although it has been reported with remarkable efficacy, it has been evidenced that the administration of chloral hydrate for sedation was not the best option in pediatric settings. ${ }^{18}$

Studies also reported that intravenous administration of propofol could be used for anesthesia purposes. It caused minimal postoperative confusion, rapid recovery and a short onset duration of 40 seconds only after administration. The medication's half-life has also been estimated to be short, as it has been estimated to be 2-4 minutes, leading to minimizing adverse events. However, it should be noted that some mild side effects were reported. These included amnesia and pain at the injection site and potentially having some antiemetic effects. In addition, reduced compliance of children remained a significant problem for administering these medications through an intravenous route. ${ }^{12,18}$ Ketamine has also been reported in these settings with a similar mechanism of action. It was primarily used for painful procedures among hemodynamically unstable patients. Ketamine, when used to induce sedation, induced catalepsy, analgesia and amnesia as the main actions of the drug in these settings. In addition, it can easily pass the bloodbrain barrier and reach the central nervous system, leading to its potential sedative effects. The peak plasma concentration of the modality had been recorded after different durations from administration, being one and five minutes following intravenous and intramuscular administration, respectively. ${ }^{12,18}$ The half-life of the drug had also been estimated to differ based on the administration route. However, evidence showed that it was metabolized more rapidly among children. Therefore, it can cause ataxia and dizziness following the administration. Moreover, it was contraindicated in cases with increased intracranial tension. ${ }^{17,22}$ This was because it can cause significant cerebral vasodilatation. ${ }^{23,24}$ Benzodiazepines were also validated in these settings, being highly selective and remarkably safe. However, it has been demonstrated that high doses can cause cardiovascular, respiratory and central nervous systems depression. They have a rapid onset of action and can be administered with midazolam, commonly performed in pediatric settings. ${ }^{18}$

\section{CONCLUSION}

Using sedation in children is growing in dentistry due to poor compliance, especially during painful procedures. In the present literature review, we have discussed sedation's different types and approaches in pediatric dentistry settings. We also reviewed the main medications that can be administered in these settings, together with the potential routes of administration, safety and contraindications. As a result, the pediatric dentist should identify patients suitable for sedation, choose the right type of sedation, prepare the media for successful procedures, and select the proper medication. Careful monitoring and adequate preparation are also critical to enhance the prognosis and manage the potential complications. Eventually, sedation might improve the compliance of pediatric patients and reduce the dilemma of fear from painful dental procedures. This has been associated with favorable outcomes for the dentist, as well. Therefore, further research is needed for additional validation and innovation in this field.

Funding: No funding sources

Conflict of interest: None declared

Ethical approval: Not required

\section{REFERENCES}

1. Edelstein B, Vargas CM, Candelaria D, Vemuri M. Experience and policy implications of children presenting with dental emergencies to US pediatric dentistry training programs. Pediatric Dentist. 2006;28(5):431-7.

2. Berg JH, Slayton RL. Early childhood oral health. John Wiley and Sons; 2015.

3. Sheiham A. Dental caries affects body weight, growth and quality of life in pre-school children. Brit Dent J. 2006;201(10):625-6.

4. Locker D, Liddell A, Dempster L, Shapiro D. Age of onset of dental anxiety. J Dent Res. 1999;78(3):790-6.

5. Milgrom P, Fiset L, Melnick S, Weinstein P. The prevalence and practice management consequences of dental fear in a major US city. J Am Dent Assoc. 1988;116(6):641-7.

6. Milgrom P, Newton JT, Boyle C, Heaton LJ, Donaldson N. The effects of dental anxiety and irregular attendance on referral for dental treatment under sedation within the national health service in London. Commun Dent Oral Epidemiol. 2010;38(5):453-9.

7. Casamassimo PS, Wilson S, Gross L. Effects of changing U.S. parenting styles on dental practice: perceptions of diplomates of the American board of pediatric dentistry presented to the college of diplomates of the American board of pediatric dentistry 16th annual session, Atlanta, Ga. Pediatric Dentist. 2002;24(1):18-22. 
8. Edelstein BL, Douglass CW. Dispelling the myth that 50 percent of U.S. schoolchildren have never had a cavity. Pub Health Rep. 1995;110(5):522-30.

9. Nelson TM, $\mathrm{Xu} \mathrm{Z}$. Pediatric dental sedation: challenges and opportunities. Clinic Cosmetic Investigat Dent. 2015;7:97-106.

10. Eslaamizaad S, Toopchi S. Sedation in Pediatric Dentistry. Acta Scientif Dent Sci. 2019;3(2).

11. Huelke DF. An overview of anatomical considerations of infants and children in the adult world of automobile safety design. Advancement Automot Med. 1998;42:93-113.

12. Appelgarth O. Understanding anesthesia: a learner's guide. Canad J Anesthes. 2013;60(9):952-3.

13. McAuliffe G, Bissonnette B, Cavallé-Garrido T, Boutin C. HR and cardiac output after atropine in anaesthetised infants and children. Canadian J Anaesthes. 1997;44:154-9.

14. Bansal T, Hooda S. Anesthetic considerations in paediatric patients. J Int Med Sci Acad. 2013;26:127-31.

15. Lourenço-Matharu L, Ashley PF, Furness S. Sedation of children undergoing dental treatment. Cochrane Database Systemat Rev. 2012;3:003877.

16. Kapur A, Kapur V. Conscious sedation in dentistry. Ann Maxillofac Surg. 2018;8(2):320-3.

17. Goodchild J, Donaldson M. The American dental association's updated sedation and general anesthesia guidelines-is minimal sedation all about triazolam? J Acad Gen Dentist. 2017;65:6.
18. Attri JP, Sharan R, Makkar V, Gupta KK, Khetarpal R, Kataria AP. Conscious sedation: emerging trends in pediatric dentistry. Anesthesia Essay Res. 2017;11(2):277-81.

19. Mennerick S, Jevtovic-Todorovic V, Todorovic SM, Shen W, Olney JW, Zorumski CF. Effect of nitrous oxide on excitatory and inhibitory synaptic transmission in hippocampal cultures. J Neurosci. 1998;18(23):9716-26.

20. Kim J, Kim S, Lee DW, Ryu DS. The alternative of oral sedation for pediatric dental care. J Dent Anesthesia Pain Med. 2015;15(1):1-4.

21. Haselkorn T, Whittemore A, Udaltsova N, Friedman G. Short-term chloral hydrate administration and cancer in humans. drug safety. Int $\mathrm{J}$ Med Toxicol Drug Exp. 2006;29:67-77.

22. Practice guidelines for intravenous conscious sedation in dentistry (Second Edition, 2017). Anesthesia Progress. 2018;65(4):1-18.

23. Kugler J, Doenicke A. Ketamine--anticonvulsive and proconvulsive actions. Der Anaesthesist. 1994;43(2):2-7.

24. Duperon DF, Jedrychowski JR. Preliminary report on the use of ketamine in pediatric dentistry. Pediatric Dent. 1983;5(1):75-8.

Cite this article as: Aljohani HR, Albalawi RA, Orri FA, Alshammari KM, Shikdar NA, Sayhab BHA, et al. Types of sedation used in pediatric dentistry. Int $\mathbf{J}$ Community Med Public Health 2022;9:466-70. 